\title{
Estrategia para el fortalecimiento del desarrollo deportivo en niños y adolescentes*
}

\author{
Luis David Martínez Quiñones ${ }^{* *}$ \\ David Felipe Zuluaga Ávila*** \\ Álvaro José Gracia Díaz ${ }^{* * * *}$
}

Recibido: 24 de enero de 2021

Aceptado: 20 de abril de 2021

Citar como:

Quiñones Martínez, L. D., Zuluaga Ávila, D. F. y Gracia Díaz, Á. J. (2021). Estrategia para el Fortalecimiento del Desarrollo Deportivo en Niños y Adolescentes.

Revista de Investigación Cuerpo, Cultura y Movimiento, 11(2).

https://doi.org/10.15332/2422474X.6761

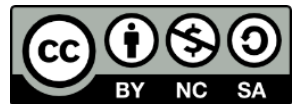

\section{Resumen}

Esta investigación propone una estrategia para fortalecer la fuerza en prepúberes, niños y adolescentes del equipo de voleibol del Colegio Pedagógico Nacional, con diferentes ejercicios dirigidos a mejorar sus diversas habilidades y a perfeccionar su desarrollo deportivo. Se utilizó el enfoque cuantitativo, la recolección de información, cuantificación y el

\footnotetext{
* Artículo de investigación. Este estudio no recibió financiación para su realización. Se realizó con el apoyo de la población del colegio Instituto Pedagógico Nacional, en Bogotá, Colombia.

** Profesional en Ciencias del Deporte de la Universidad de Ciencias Aplicadas y Ambientales, U.D.C.A. Correo electrónico: tettomartinez@hotmail.com; ORCID: https://orcid.org/0000-00016660-8260

*** Profesional en Ciencias del Deporte de la Universidad de Ciencias Aplicadas y Ambientales, U.D.C.A. Correo electrónico: davidfpezuluaga@gmail.com; ORCID: https://orcid.org/0000-00017389-0022

**** Dr. (Ph. D.) en Pedagogía y Didáctica de la Educación Física en Kiev, Ucrania. Correo electrónico: algradi333@hotmail.com, agracia@udca.edu.co; ORCID: https://orcid.org/00000001-7572-145X
}

Revista de Investigación Cuerpo, Cultura y Movimiento

ISSN: 2248-4418 | e-ISSN: 2422-474X | DOI: https://doi.org/10.15332/2422474X

Vol. 11 N.0 2 | julio-diciembre de 2021 
análisis estadístico, dirigidos a fomentar coordenadas de actuación y a comprobar la hipótesis de que la fuerza en la población estudiada crece en línea ascendente al incorporar una estrategia apropiada del entrenamiento de la fuerza. El diseño transversal y la manera correlacional hallaron el vínculo entre dos o más variables implicadas. Los resultados del ejercicio investigativo demostraron que la implementación de un proceso de manejo de fuerza con carga baja y un aumento ascendente genera beneficios en los niños y los jóvenes para mejorar su forma de actuación y su estado físico.

Palabras clave: prepúberes, programa de entrenamiento, entrenamiento fuerza, desarrollo deportivo.

\section{Strategy for strengthening sports development in children and adolescents}

\section{Abstract}

This research seeks a strategy to improve strength in prepubescent, children and adolescents of the volleyball team of the Colegio Pedagógico Nacional, with different exercises aimed at improving their different skills and perfecting their sports development. The quantitative approach, data collection, quantification and statistical analysis were used, aimed at promoting performance patterns and testing the hypothesis that strength in the studied population increases in an ascending line when incorporating an appropriate strategy of strength training. The cross-sectional design and correlational manner found the relation between two or more variables involved. The results of the research exercise showed that the implementation of a strength management program with a low load and a progressive increase produces benefits in children and young people to improve their performance and physical condition.

Keywords: prepubescent, training program, strength training, sports development. 


\section{Introducción}

La presente investigación reconoció los impactos, favorables y desfavorables, en niños y jóvenes de diez a trece años del equipo de voleibol del colegio Instituto Pedagógico Nacional e identificó los avances obtenidos en la introducción de la estrategia para el fortalecimiento de la fuerza en prepúberes. Para ello se tuvo en cuenta el consenso internacional realizado en el año 2014, por varios autores como Lloyd, Faigenbaum, Stone y Oliver (2014). En este estudio se constata el crecimiento de la cooperación de niños y jóvenes en variadas formas de abordaje de la fuerza y también el aumento de investigadores, profesionales en salud y entrenadores profesionales sobre este tema. Es importante resaltar que la investigación se llevó a cabo de forma correlacional, pues se buscaba hallar la relación entre dos o más variables con el fin de pronosticar una actuación a largo plazo (Hernández et ál., 2014).

De la misma manera, en la investigación de Behringer et ál. (2011) se evidencia los niveles de progreso de los prepúberes, y se permite la identificación del avance en el rendimiento motriz a través del aumento de fuerza, celeridad, potencia, entre otras.

Los antecedentes tienen sus cimientos en los 50 artículos funcionales encontrados en las diferentes bases de datos a nivel internacional. Esta búsqueda se realizó a través de cuatro palabras clave: entrenamiento, fuerza, edades tempranas y desarrollo deportivo a través de ProQuest (5), Dialnet (10), G-SE (24), Google Académico (10) y PubMed (1). Son artículos publicados en inglés y español desde los años 80 del siglo pasado hasta el 2016 en reconocidas revistas (tabla 1).

La anterior exploración nos condujo a constatar la insuficiencia de referencias acerca del entrenamiento de la fuerza en jóvenes a nivel 
nacional y vislumbró la necesidad de realizar una investigación para conocer las consecuencias del entrenamiento de la fuerza a temprana edad, siendo una situación oportuna para abordar este importante tema.

Continuando con la exploración de las fuentes, encontramos a Faigembaum (2000) indica la relevancia de conocer los efectos de la fuerza a lo largo del crecimiento y el desarrollo de prepúberes y señala que por el momento no existe evidencia científica acerca de qué una actividad física específica pueda perturbar una normal evolución de tipo psíquico, físico, emotivo y cognitivo. Sin embargo, Winter (1986) afirma que las capacidades no son entrenables de la misma forma en cada una de las edades. Adicionalmente, Beunen y Malina (2008) afirman que la evolución, desarrollo y maduración son términos empleados para explicar variaciones físicas desde la niñez hasta la adultez.

Ahora bien, es necesario tener en cuenta que, de acuerdo con Grosser, Brüggemann y Zintl (1989), la evolución orgánica, es decir, el desarrollo de las capacidades a nivel de coordinación y condicionales en prepúberes, presenta características específicas; por ejemplo, las actividades elementales de coordinación se gestan desde los 6 años y logran su cumbre a los 12 años, aproximadamente. De igual forma, alrededor de los 12 años comienzan las etapas sensibles, en las que se desenvuelven y prosperan las habilidades para la condición física.

Teniendo en cuenta lo anterior, es fundamental aludir a Martín, citado por Hahn (1988), quien investiga la relación existente entre las condiciones psicomotrices de una persona y el nivel de desarrollo esperado según su edad. Este llega a la conclusión de que el individuo presenta un desarrollo superior de sus capacidades en ciclos determinados.

Armstrong y Mechelen (2013) demuestran que el desarrollo es un elemento que involucra varios aspectos que dependen tanto de la 
condición física como de la unión de factores musculares, neurológicos y biomecánicos. De igual forma, Faigenbaum et ál. (2015) recomiendan calcular adecuadamente el entrenamiento de la fuerza.

A pesar de que se pensaba que la preparación física en prepúberes traía efectos adversos, muchos estudios, como Age changes in motor skills during childhoob and adolescence, realizado por Branta, Haubenstricker y Seefeldt (1984) muestran cómo la fuerza muscular incrementa de manera aproximadamente lineal durante la niñez y adolescencia, tanto en mujeres como en hombres. De igual forma, Malina y Bouchard (2004) ilustran cómo el adiestramiento de la fuerza, en la adolescencia, fomenta el desarrollo neuronal y en la testosterona.

Otro aspecto importante lo observaron, Martin et ál. (1991), Asmus (1991) y Martin (1982), que hallaron conexiones entre competencias como: respuesta, rapidez, fortaleza anaeróbica y aeróbica, fuerza cumbre, equilibrio, obtención de habilidades y técnicas en la infancia y la adolescencia.

Además, en los últimos años, la Organización Mundial de la Salud (2020) realizó recomendaciones respecto a la actividad física. También evidencia que la ausencia de ejercicio físico se considera como una de las causas de muerte a nivel planetario en enfermedades comunes. Por lo que favorece la participación de toda la población en variadas actividades, incluyendo aquellas que fortalecen los músculos y los huesos. Enfatiza que realizar entrenamiento físico y deporte produce impactos positivos en la salud física, mental y social de quienes la practican.

Otra variable importante tiene que ver con la cumbre de obtención de fuerza, particularmente en infantes, conectada con el momento más importante del desarrollo para el entrenamiento de la fuerza muscular. Esta última se da, aproximadamente, a los doce años posterior a la cúspide 
de la máxima estatura y 0.8 años antes que la cima de velocidad de incremento de peso. Al mismo tiempo, el desarrollo de la aptitud de fuerza ingresa en un periodo sensible que inicia ad portas de la pubertad, en las niñas, generalmente, desde los 11 años, y en los niños, a comienzos de los 12 años (Faigembaum et ál., 1996).

Así mismo, la capacidad de fuerza rápida se puede elevar eficientemente y con toda certeza finalizando la edad escolar primaria y a inicios de la secundaria (Winter, 1986). En la infancia, para realizar actividad física con frecuencia, necesita ser proyectada e inspeccionada; además, requiere tener principalmente juego en el que se aprenda y descubra movimientos cada vez más complicados (Faigenbaum, Milliken y Cloutier, 2004; Faigembaum et ál. 1996).

De acuerdo con lo anterior, y en un ámbito educativo, el instructor o docente necesitará organizar los entrenamientos modificando el volumen y la intensidad a lo largo del periodo, de tal forma que pueda ir cumpliendo los objetivos para aumentar la complejidad de los ejercicios de forma escalonada conforme la capacidad específica de cada niño y adolescente (Bompa, 2003).

Complementando al anterior autor, Faigembaum et ál. (1996) propone tres objetivos esenciales para el entrenamiento en la primera infancia:

- Ayudar al crecimiento y desarrollo.

- Optimizar el rendimiento físico y deportivo.

- $\quad$ Prever las lesiones brindando mayor resistencia y estabilidad en los tejidos blandos, óseos y masa muscular.

- $\quad$ tejidos blandos, óseos y masa muscular.

Además de considerar la constitución corporal, el panel de grasas, la densidad ósea, la capacidad motriz general y factores psicológicos o actitudinales relacionados con la propensión para elegir actividades con 
mayor necesidad de energía, como las deportivas, también es necesario compararlas en contraposición con otras actividades sedentarias, tales como estar en el computador, mirar televisión, etc. (Watts et ál., 2005). Faigembaum et ál. (1996) analizaron a niños entre los 7 y 12 años, que iniciaron una fase de entrenamiento de fuerza con resistencias, dos veces, de lunes a domingo. Entre las primeras cuatro semanas realizaron un par de tandas con 6 y 8 reiteraciones en cada actividad y en las siguientes semanas aumentaron a tres series. Posteriormente, seguían con una fase de ocho semanas en las que no había ningún entrenamiento de fuerza, llamado como el periodo de desentrenamiento. Los hallazgos de esta investigación arrojaron que en la población estudiada aumentaba de una manera importante el grado de fuerza cumbre en la actividad de estiramiento de pierna, al tiempo que esto ocurría con el grupo control. Aunque revelaba notorios avances frente a los datos primarios, los avances eran menores a los del grupo experimental.

Otra de las conclusiones de este estudio fue que las instrucciones, dadas por los acudientes de los infantes que participaban, lograban mejorar su ejecución en las competencias o en los eventos deportivos concretos, así como su atención para adelantar las jornadas de preparación rutinarias. Así mismo, el fin primordial del entrenamiento deportivo en niños es lograr un nivel cumbre de experiencia motriz en la fortaleza, la resistencia y la rapidez en ejercicios en los que el infante aprenda de manera progresiva, de tal forma que no sean demasiado complejas para su capacidad (Martin, 1982).

Finalmente, vale la pena decir que una buena parte de los autores coincide en que el entrenamiento físico y deportivo desde temprana edad impacta positivamente en el desarrollo de las capacidades futuras de esta población. Ahora bien, es necesario poner a prueba nuestra hipótesis 
respecto a la fuerza en los jóvenes crece en forma ascendente y sostenida al incorporar un programa de entrenamiento de fuerza apropiado. Para esto, de manera inicial, se realizó una revisión bibliográfica y una búsqueda organizada de producción de textos en las fuentes arriba mencionadas (tabla 1).

Tabla 1. Resultados de la búsqueda de artículos

\begin{tabular}{|c|c|c|c|c|}
\hline Base Datos & Idioma & Año & N. ${ }^{\circ}$ Artículos & Funcionales \\
\hline Google Académico: & Español. Inglés. & $1990-2016$ & 10 & 10 \\
\hline Dialnet: & Inglés. & $1990-2015$ & 10 & 10 \\
\hline Proquest: & Español. Inglés. & $1990-2015$ & 5 & 5 \\
\hline G-Se: & Español. Inglés. & $1990-2015$ & 24 & 24 \\
\hline Pubmed: & Español. Inglés. & $1990-2016$ & 1 & 1 \\
\hline Total encontrados: & - & - & 50 & 50 \\
\hline
\end{tabular}

Fuente: elaboración propia.

Es importante mencionar que se entienden como funcionales todos los artículos que contenían información sobre el impacto positivo de la fuerza, programas de entrenamiento, preparación en prepúberes y efectos de costumbres repetitivas en niños y adolescentes.

\section{Enfoque e instrumentos}

Este estudio se trabajó desde la visión cuantitativa, que permitió ejecutar la obtención de información para demostrar la hipótesis a través de la cuantificación y el análisis estadístico, con el fin de determinar modelos de comportamiento y comprobar teorías. De igual forma, la investigación aplicó el diseño transversal y se centró en establecer hasta dónde se había avanzado en el presente tema. La técnica de recolección de datos se realizó en dos momentos específicos y se ubicó dentro del corte descriptivocorrelacional, debido a que se buscaba medir y reunir datos acerca de los 
miembros del equipo de voleibol, se consideró el nivel de vinculación que existía entre las variables de la investigación (Hernández et ál., 2014).

\section{Población}

Se eligió un grupo de prepúberes de (10 a 13 años) de la institución donde se desarrolló este estudio, que hicieran parte del equipo de voleibol.

\section{Muestra}

Se seleccionó, a través en un muestreo aleatorio, a 19 prepúberes pertenecientes al equipo de voleibol del colegio Instituto Pedagógico Nacional, entre los 10 y 13 años.

\section{Instrumentos y herramientas}

De manera inicial se estableció un cronograma de entrenamiento, de ocho semanas, con el objetivo de planificar las actividades y comprobar o no la hipótesis propuesta, como se evidencia en la tabla 2.

Posteriormente se construye el diagrama de Gantt con el objetivo de visualizar el tiempo que se invertirá en cada una de las actividades, como se observa en la tabla 3 .

En la tabla 4 se evidencia el macrociclo de la estrategia de abordaje de la fuerza: 
Tabla 2. Cronograma del entrenamiento con la población estudiada

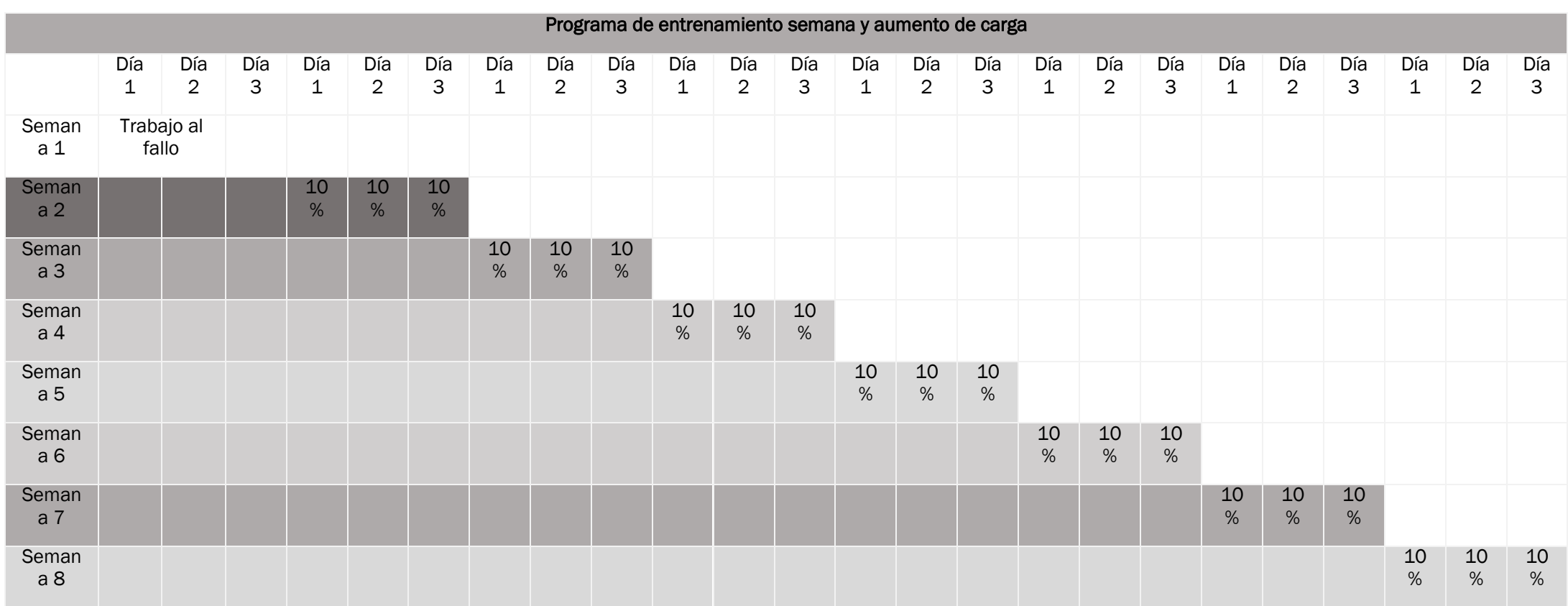

Fuente: elaboración propia.

Revista de Investigación Cuerpo, Cultura y Movimiento

ISSN: 2248-4418 | e-ISSN: 2422-474X | DOI: https://doi.org/10.15332/2422474X

Vol. 11 N.0 2 | julio-diciembre de 2021 
Tabla 3. Esquema de Gantt

\begin{tabular}{|c|c|c|c|c|c|c|c|c|c|c|c|}
\hline \multicolumn{3}{|c|}{ Macrociclo } & \multicolumn{9}{|c|}{ Entrenamiento de la fuerza pre-púberes } \\
\hline \multicolumn{3}{|c|}{ Fase de la forma deportiva } & \multicolumn{9}{|c|}{ Fase de adquisición } \\
\hline \multicolumn{3}{|c|}{ Periodo de preparación } & \multicolumn{9}{|c|}{ Periodo preparatorio } \\
\hline \multicolumn{3}{|c|}{ Etapas de preparación } & \multicolumn{9}{|c|}{ Preparación general } \\
\hline \multicolumn{3}{|c|}{ Mesociclo } & Entrante & \multicolumn{4}{|c|}{ Desarrollador } & \multicolumn{4}{|c|}{ Estabilizador } \\
\hline \multicolumn{3}{|c|}{ Meses } & \multicolumn{5}{|c|}{ Octubre } & \multicolumn{4}{|c|}{ Noviembre } \\
\hline \multicolumn{3}{|c|}{ Semanas } & 1 & 2 & 3 & 4 & 5 & 6 & 7 & 8 & 9 \\
\hline \multirow{2}{*}{\multicolumn{2}{|c|}{ Fechas }} & DEL & 29 & 6 & 13 & 20 & 27 & 3 & 10 & 17 & 24 \\
\hline & & $\mathrm{AL}$ & 5 & 12 & 19 & 26 & 2 & 9 & 16 & 23 & 30 \\
\hline \multicolumn{3}{|c|}{ Tipos de microciclos } & C & C & C & C & C & C & C & C & C \\
\hline \multicolumn{3}{|c|}{ Sesiones } & 3 & 3 & 3 & 3 & 3 & 3 & 3 & 3 & 3 \\
\hline \multicolumn{3}{|c|}{ Tiempo real periodo } & \multicolumn{9}{|c|}{2025} \\
\hline \multicolumn{3}{|c|}{ Tiempo planificado periodo } & \multicolumn{9}{|c|}{1620} \\
\hline \multicolumn{3}{|c|}{ Tiempo real etapa } & \multicolumn{9}{|c|}{2025} \\
\hline \multicolumn{3}{|c|}{ Tiempo planificado etapa } & \multicolumn{9}{|c|}{1620} \\
\hline \multicolumn{3}{|c|}{ Tiempo real mesociclo } & 225 & \multicolumn{4}{|c|}{900} & \multicolumn{4}{|c|}{900} \\
\hline \multicolumn{3}{|c|}{ Tiempo planificado mesociclo } & 180 & \multicolumn{4}{|c|}{720} & & & & \\
\hline & Tiempo real microciclo & & 225 & 225 & 225 & 225 & 225 & 225 & 225 & 225 & 225 \\
\hline & Tiempo planificado microciclo & & 180 & 180 & 180 & 180 & 180 & 180 & 180 & 180 & 180 \\
\hline & Tiempo real sesión & & 75 & 75 & 75 & 75 & 75 & 75 & 75 & 75 & 75 \\
\hline & Tiempo planificado sesión & & 60 & 60 & 60 & 60 & 60 & 60 & 60 & 60 & 60 \\
\hline Componentes & Dirección & Métodos & & & & & & & & & \\
\hline & Preparación física general & & & & & & & & & & \\
\hline Fuerza & Adaptación Anatómica & Autocarga & $20 \% / 324$ & & & & & & & & \\
\hline
\end{tabular}

Revista de Investigación Cuerpo, Cultura y Movimiento

ISSN: 2248-4418 | e-ISSN: 2422-474X | DOI: https://doi.org/10.15332/2422474X

Vol. 11 N.o 2 | julio-diciembre de 2021 


\begin{tabular}{|c|c|c|c|}
\hline \multicolumn{1}{|c|}{ Macrociclo } & & Entrenamiento de la fuerza pre-púberes \\
\hline Fuerza Resistencia & Autocarga & \\
\hline Fuerza Explosiva & Autocarga & \\
\hline Fuente: elaboración propia. & & \\
\hline
\end{tabular}

Tabla 4. Macrociclo entrenamiento de la fuerza prepúberes

\begin{tabular}{|c|c|c|c|c|c|c|c|c|c|c|}
\hline \multicolumn{2}{|c|}{ Macrociclo } & \multicolumn{9}{|c|}{ Entrenamiento de la fuerza pre-púberes } \\
\hline \multicolumn{2}{|c|}{ Fase de la forma deportiva } & \multicolumn{9}{|c|}{ Fase de adquisición } \\
\hline \multicolumn{2}{|c|}{ Periodo de preparación } & \multicolumn{9}{|c|}{ Periodo preparatorio } \\
\hline Etapas de & & \multicolumn{9}{|c|}{ Preparación general } \\
\hline \multicolumn{2}{|c|}{ Mesociclo } & Entrante & \multicolumn{4}{|c|}{ Desarrollador } & \multicolumn{4}{|c|}{ Estabilizador } \\
\hline \multicolumn{2}{|c|}{ Meses } & \multicolumn{5}{|c|}{ Octubre } & \multicolumn{4}{|c|}{ Noviembre } \\
\hline \multicolumn{2}{|c|}{ Semanas } & 1 & 2 & 3 & 4 & 5 & 6 & 7 & 8 & 9 \\
\hline \multirow{2}{*}{ Fechas } & DEL & 29 & 6 & 13 & 20 & 27 & 3 & 10 & 17 & 24 \\
\hline & AL & 5 & 12 & 19 & 26 & 2 & 9 & 16 & 23 & 30 \\
\hline \multicolumn{2}{|c|}{ Tipos de microciclos } & C & C & C & C & C & C & C & C & C \\
\hline \multicolumn{2}{|c|}{ Sesiones } & 3 & 3 & 3 & 3 & 3 & 3 & 3 & 3 & 3 \\
\hline \multicolumn{2}{|c|}{ Tiempo real periodo } & \multicolumn{9}{|c|}{2025} \\
\hline \multicolumn{2}{|c|}{ Tiempo planificado periodo } & \multicolumn{9}{|c|}{1620} \\
\hline \multicolumn{2}{|c|}{ Tiempo real etapa } & \multicolumn{9}{|c|}{2025} \\
\hline \multicolumn{2}{|c|}{ Tiempo planificado etapa } & \multicolumn{9}{|c|}{1620} \\
\hline \multicolumn{2}{|c|}{ Tiempo real mesociclo } & 225 & \multicolumn{4}{|c|}{900} & \multicolumn{4}{|c|}{900} \\
\hline \multicolumn{2}{|c|}{ Tiempo planificado mesociclo } & 180 & \multicolumn{4}{|c|}{720} & \multicolumn{4}{|c|}{720} \\
\hline \multicolumn{2}{|c|}{ Tiempo real microciclo } & 225 & 225 & 225 & 225 & 225 & 225 & 225 & 225 & 225 \\
\hline \multicolumn{2}{|c|}{ Tiempo planificado microciclo } & 180 & 180 & 180 & 180 & 180 & 180 & 180 & 180 & 180 \\
\hline
\end{tabular}

Revista de Investigación Cuerpo, Cultura y Movimiento

ISSN: 2248-4418 | e-ISSN: 2422-474X | DOI: https://doi.org/10.15332/2422474X

Vol. 11 N.O 2 | julio-diciembre de 2021 
Entrenamiento de la fuerza pre-púberes

\begin{tabular}{|c|c|c|c|c|c|c|c|c|c|c|c|}
\hline \multicolumn{3}{|c|}{ Macrociclo } & \multicolumn{9}{|c|}{ Entrenamiento de la fuerza pre-púberes } \\
\hline \multicolumn{3}{|c|}{ Tiempo real sesión } & 75 & 75 & 75 & 75 & 75 & 75 & 75 & 75 & 75 \\
\hline \multicolumn{3}{|c|}{ Tiempo planificado sesión } & 60 & 60 & 60 & 60 & 60 & 60 & 60 & 60 & 60 \\
\hline Componentes & Dirección & Métodos & & & & & & & & & \\
\hline \multicolumn{12}{|c|}{ Preparación física general } \\
\hline \multirow{3}{*}{ Fuerza } & Adaptación anatómica & Autocarga & & & & & & & & & \\
\hline & Fuerza resistencia & Autocarga & & & & & & & & & \\
\hline & Fuerza explosiva & Autocarga & & & & & & & & & \\
\hline
\end{tabular}

Fuente: elaboración propia.

Revista de Investigación Cuerpo, Cultura y Movimiento

ISSN: 2248-4418 | e-ISSN: 2422-474X | DOI: https://doi.org/10.15332/2422474X

Vol. 11 N.0 2 | julio-diciembre de 2021 
También, para observar el mesociclo de la fase entrante o inicial, se puede ver la tabla 5 .

Tabla 5. Mesociclo-fase entrante

\begin{tabular}{|c|c|c|c|}
\hline \multicolumn{3}{|c|}{ Etapas de preparación } & PP \\
\hline \multicolumn{3}{|c|}{ Mesociclo } & Entrante \\
\hline \multicolumn{3}{|c|}{ Meses } & Octubre \\
\hline \multicolumn{3}{|c|}{ Semanas } & 1 \\
\hline \multirow{2}{*}{\multicolumn{2}{|c|}{ Fechas }} & DEL & 29 \\
\hline & & $\mathrm{AL}$ & 5 \\
\hline & os de microciclos & & C \\
\hline \multicolumn{3}{|c|}{ Sesiones } & 3 \\
\hline \multicolumn{3}{|c|}{ Tiempo real periodo } & 225 \\
\hline \multicolumn{3}{|c|}{ Tiempo planificado periodo } & 180 \\
\hline \multicolumn{3}{|c|}{ Tiempo real etapa } & 225 \\
\hline \multicolumn{3}{|c|}{ Tiempo planificado etapa } & 180 \\
\hline \multicolumn{3}{|c|}{ Tiempo real mesociclo } & 225 \\
\hline \multicolumn{3}{|c|}{ Tiempo planificado mesociclo } & 180 \\
\hline \multicolumn{3}{|c|}{ Tiempo real microciclo } & 225 \\
\hline \multicolumn{3}{|c|}{ Tiempo planificado microciclo } & 180 \\
\hline \multicolumn{3}{|c|}{ Tiempo real sesión } & 75 \\
\hline \multicolumn{3}{|c|}{ Tiempo planificado sesión } & 60 \\
\hline \multirow[t]{2}{*}{ Componentes } & Dirección & Métodos & \\
\hline & \multicolumn{2}{|c|}{ Preparación física general } & \\
\hline \multirow{3}{*}{ Fuerza } & Adaptación anatómica & Autocarga & 60 \\
\hline & Fuerza resistencia & Autocarga & \\
\hline & Fuerza explosiva & Autocarga & \\
\hline
\end{tabular}

Fuente: elaboración propia.

Al mismo tiempo se observa el mesociclo, pero esta vez en la fase desarrollador (tabla 6). 
Tabla 6. Mesociclo-fase desarrollador

\begin{tabular}{|c|c|c|c|c|c|c|}
\hline \multicolumn{3}{|c|}{ Etapas de preparación } & \multicolumn{4}{|c|}{ Preparación General } \\
\hline \multicolumn{3}{|c|}{ Mesociclo } & \multicolumn{4}{|c|}{ Desarrollador } \\
\hline \multicolumn{3}{|c|}{ Meses } & \multicolumn{4}{|c|}{ Octubre } \\
\hline \multicolumn{3}{|c|}{ Semanas } & 2 & 3 & 4 & 5 \\
\hline \multirow{2}{*}{\multicolumn{2}{|c|}{ Fechas }} & DEL & 6 & 13 & 20 & 27 \\
\hline & & $\mathrm{AL}$ & 12 & 19 & 26 & 2 \\
\hline \multicolumn{3}{|c|}{ Tipos de microciclos } & C & C & C & C \\
\hline \multicolumn{3}{|c|}{ Sesiones } & 3 & 3 & 3 & 3 \\
\hline \multicolumn{3}{|c|}{ Tiempo real periodo } & \multicolumn{4}{|c|}{900} \\
\hline \multicolumn{3}{|c|}{ Tiempo planificado periodo } & \multicolumn{4}{|c|}{720} \\
\hline \multicolumn{3}{|c|}{ Tiempo real etapa } & \multicolumn{4}{|c|}{900} \\
\hline \multicolumn{3}{|c|}{ Tiempo planificado etapa } & \multicolumn{4}{|c|}{720} \\
\hline \multicolumn{3}{|c|}{ Tiempo real mesociclo } & \multicolumn{4}{|c|}{900} \\
\hline \multicolumn{3}{|c|}{ Tiempo planificado mesociclo } & \multicolumn{4}{|c|}{720} \\
\hline \multicolumn{3}{|c|}{ Tiempo real microciclo } & 225 & 225 & 225 & 225 \\
\hline \multicolumn{3}{|c|}{ Tiempo planificado microciclo } & 180 & 180 & 180 & 180 \\
\hline \multicolumn{3}{|c|}{ Tiempo real sesión } & 75 & 75 & 75 & 75 \\
\hline \multicolumn{3}{|c|}{ Tiempo planificado sesión } & 60 & 60 & 60 & 60 \\
\hline Componentes & Dirección & Métodos & & & & \\
\hline \multicolumn{7}{|c|}{ Preparación física general } \\
\hline \multirow{3}{*}{ Fuerza } & Adaptación anatómica & Autocarga & & & & \\
\hline & Fuerza resistencia & Autocarga & 60 & 60 & 60 & 60 \\
\hline & Fuerza explosiva & Autocarga & & & & \\
\hline
\end{tabular}

Fuente: elaboración propia.

Finalmente, se elabora y presenta el mesociclo en la fase estabilizador, como se observa en la tabla 7. 
Tabla 7. Mesociclo-fase estabilizador

\begin{tabular}{|c|c|c|c|c|c|c|}
\hline \multicolumn{3}{|c|}{ Etapas de preparación } & & & & \\
\hline \multicolumn{3}{|c|}{ Mesociclo } & \multicolumn{4}{|c|}{ Estabilizador } \\
\hline \multicolumn{3}{|c|}{ Meses } & \multicolumn{4}{|c|}{ Noviembre } \\
\hline \multicolumn{3}{|c|}{ Semanas } & 6 & 7 & 8 & 9 \\
\hline \multirow{2}{*}{\multicolumn{2}{|c|}{ Fechas }} & DEL & 3 & 10 & 17 & 24 \\
\hline & & $\mathrm{AL}$ & 9 & 16 & 23 & 30 \\
\hline \multicolumn{3}{|c|}{ Tipos de microciclos } & C & C & C & C \\
\hline \multicolumn{3}{|c|}{ Sesiones } & 3 & 3 & 3 & 3 \\
\hline \multicolumn{3}{|c|}{ Tiempo real periodo } & \multicolumn{4}{|c|}{900} \\
\hline \multicolumn{3}{|c|}{ Tiempo planificado periodo } & \multicolumn{4}{|c|}{720} \\
\hline \multicolumn{3}{|c|}{ Tiempo real etapa } & \multicolumn{4}{|c|}{900} \\
\hline \multicolumn{3}{|c|}{ Tiempo planificado etapa } & \multicolumn{4}{|c|}{720} \\
\hline \multicolumn{3}{|c|}{ Tiempo real mesociclo } & \multicolumn{4}{|c|}{900} \\
\hline \multicolumn{3}{|c|}{ Tiempo planificado mesociclo } & \multicolumn{4}{|c|}{720} \\
\hline \multicolumn{3}{|c|}{ Tiempo real microciclo } & 225 & 225 & 225 & 225 \\
\hline \multicolumn{3}{|c|}{ Tiempo planificado microciclo } & 180 & 180 & 180 & 180 \\
\hline \multicolumn{3}{|c|}{ Tiempo real sesión } & 75 & 75 & 75 & 75 \\
\hline \multicolumn{3}{|c|}{ Tiempo planificado sesión } & 60 & 60 & 60 & 60 \\
\hline \multirow{2}{*}{\multicolumn{3}{|c|}{$\begin{array}{l}\text { Componentes Dirección } \\
\text { Preparación física general }\end{array}$}} & & & & \\
\hline & & & & & & \\
\hline \multirow{3}{*}{ Fuerza } & Adaptación anatómica & Autocarga & & & & \\
\hline & Fuerza resistencia & Autocarga & & & & \\
\hline & Fuerza explosiva & Autocarga & 60 & 60 & 60 & 60 \\
\hline
\end{tabular}

Fuente: elaboración propia.

\section{Consejos para la ejecución de un programa de entrenamiento en fuerza}

1. Mantener el número total de participantes a lo largo de todo el proceso y ejercicios a realizar.

2. Es importante la participación de un psicólogo del deporte en todo el proceso, con el fin de solucionar las eventualidades emocionales que puedan llegar a afectar el rendimiento deportivo y la deserción. 
3. Se requiere la vigilancia de un nutricionista en la alimentación de los participantes, lo que permite una recuperación muscular más eficiente y evitar la alteración en el rendimiento físico y por ende en los resultados.

4. Comprometer a los participantes y deportistas durante todo el proceso práctico.

5. Construir una metodología innovadora para reducir la deserción de los participantes.

6. Realizar actividades que incrementen el interés de infantes y jóvenes durante el programa de entrenamiento.

7. Ofrecer definiciones teóricas acerca de los impactos favorables y objetivos que se quieren lograr con los participantes.

\section{Resultados y discusión}

De acuerdo con la tabla 2, que evidencia el tiempo de trabajo durante ocho semanas, y la puesta en práctica de una estrategia de abordaje de la fuerza, se observa la actividad dosificada según las necesidades. Así, durante los primeros días, en el día 1, la actividad adelantada con los niños y adolescentes fue al fallo.

\section{Comparación antes y después de la prueba, flexiones de rodilla}

En la figura 1 se observan los contrastes en las distintas prácticas de pruebas de rodilla antes y después de la prueba. Esto nos permite evidenciar un progreso posterior a la aplicación de la estrategia de abordaje de la fuerza: cada persona mejoró su marcador anterior, a excepción del individuo 15, que no tuvo variación entre el pre y postest. Lo anterior significa que, aunque hubo una mayor calidad en el movimiento, no lo hubo en el número de repeticiones, por ende, no existió mejora (figura 1). 
Figura 1. Repeticiones pre- y postest de flexiones de rodilla

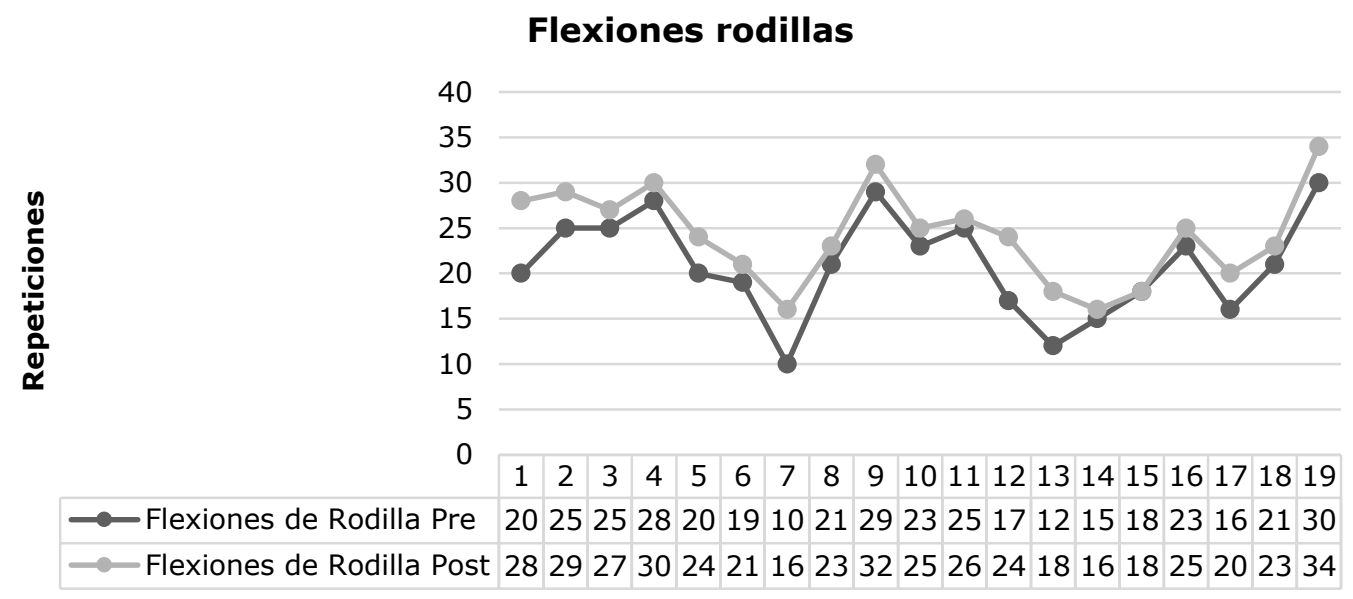

Individuos

Fuente: elaboración propia.

A continuación, se observa en la tabla 8 el mejoramiento en las flexiones de rodilla; la persona con menor porcentaje de progreso fue el número 15, que obtuvo cero por ciento (o \%). Así mismo, el individuo más sobresaliente fue el número (7) con el sesenta por ciento (6o \%).

Tabla 7. Porcentaje de mejora flexiones de rodilla

\begin{tabular}{|c|c|c|c|}
\hline Individuos & Flexiones rodillas & Individuos & Flexiones rodillas \\
\hline 1 & 40 & 11 & 4 \\
\hline 2 & 16 & 12 & 41 \\
\hline 3 & 8 & 13 & 50 \\
\hline 4 & 7 & 14 & 7 \\
\hline 5 & 20 & 15 & 0 \\
\hline 6 & 11 & 16 & 9 \\
\hline 7 & 60 & 17 & 25 \\
\hline 9 & 10 & 18 & 10 \\
\hline 10 & 10 & 19 & 13 \\
\hline
\end{tabular}

Fuente: elaboración propia.

Revista de Investigación Cuerpo, Cultura y Movimiento ISSN: 2248-4418 | e-ISSN: 2422-474X | DOI: https://doi.org/10.15332/2422474X Vol. 11 N. ${ }^{\circ} 2$ | julio-diciembre de 2021 
En la figura 2, se muestra el contraste entre las rutinas realizadas del pretest y el postest, y el progreso que todos obtuvieron posterior a haber ejecutado la estrategia de abordaje de la fuerza; también, los evaluadores evidenciaron una mejoría en la calidad del ejercicio realizado, reflejados en el postest con las secuencias (figura 2).

Figura 2. Repeticiones antes y después de la prueba flexiones sentadillas

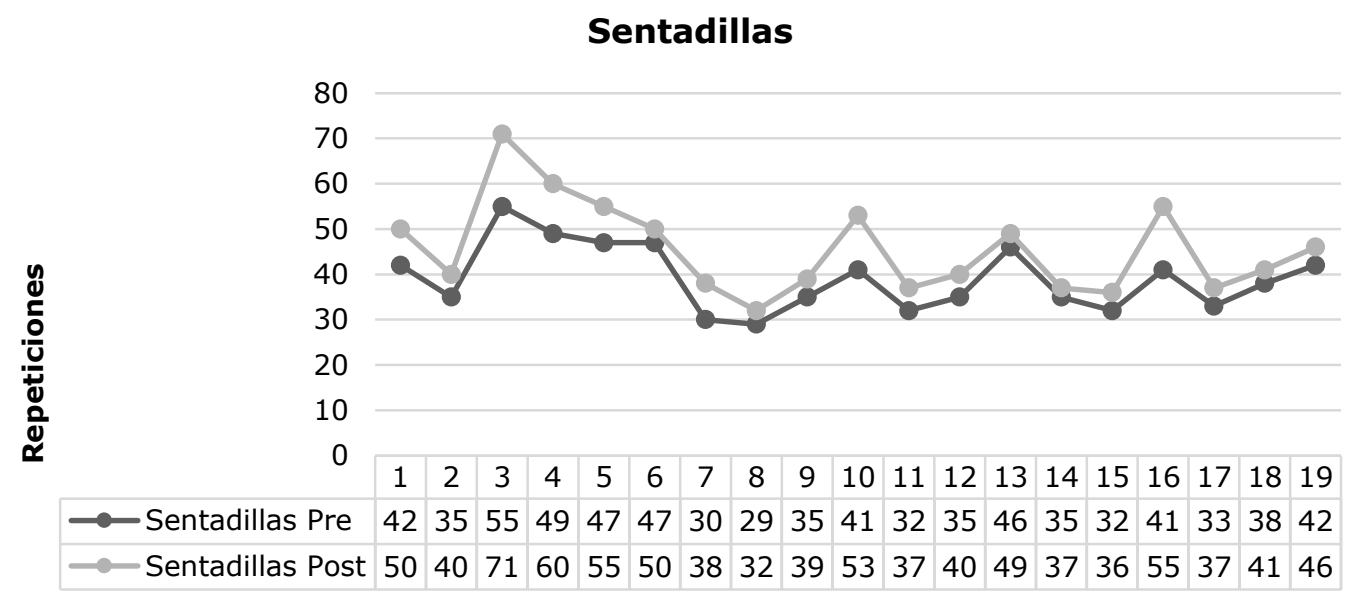

Individuos

Fuente: elaboración propia.

De acuerdo con la tabla 9, se aprecia la actividad ejecutada por los participantes antes y después de iniciar la estrategia de abordaje de la fuerza. Por ejemplo, el individuo 8 realizó 29 secuencias de sentadilla en el pretest y 32 secuencias en el postest (tabla 3 ).

Tabla 8. Comparación trabajo al fallo antes y después de la prueba de sentadilla.

\begin{tabular}{|c|c|c|c|}
\hline \multicolumn{2}{|c|}{ Pretest } & & \multicolumn{2}{c|}{ Postest } \\
\hline Individuos & Sentadillas & Individuos & Sentadillas \\
\hline 19 & 744 & 19 & 866 \\
\hline Promedio & 39 & Promedio & 46 \\
\hline
\end{tabular}

Fuente: elaboración propia. 
En la figura 3, se puede observar el contraste entre las secuencias ejecutadas en el pretest y el postest. Se concluye que cada uno de los participantes obtuvo una mejora notable posterior a la aplicación del programa de entrenamiento en fuerza, a pesar del aumento mínimo de algunos individuos, lo que puede destacarse es que en todo momento hubo un progreso entre el antes y el después (figura 3).

Figura 3. Repeticiones antes y después de la prueba de abdominales

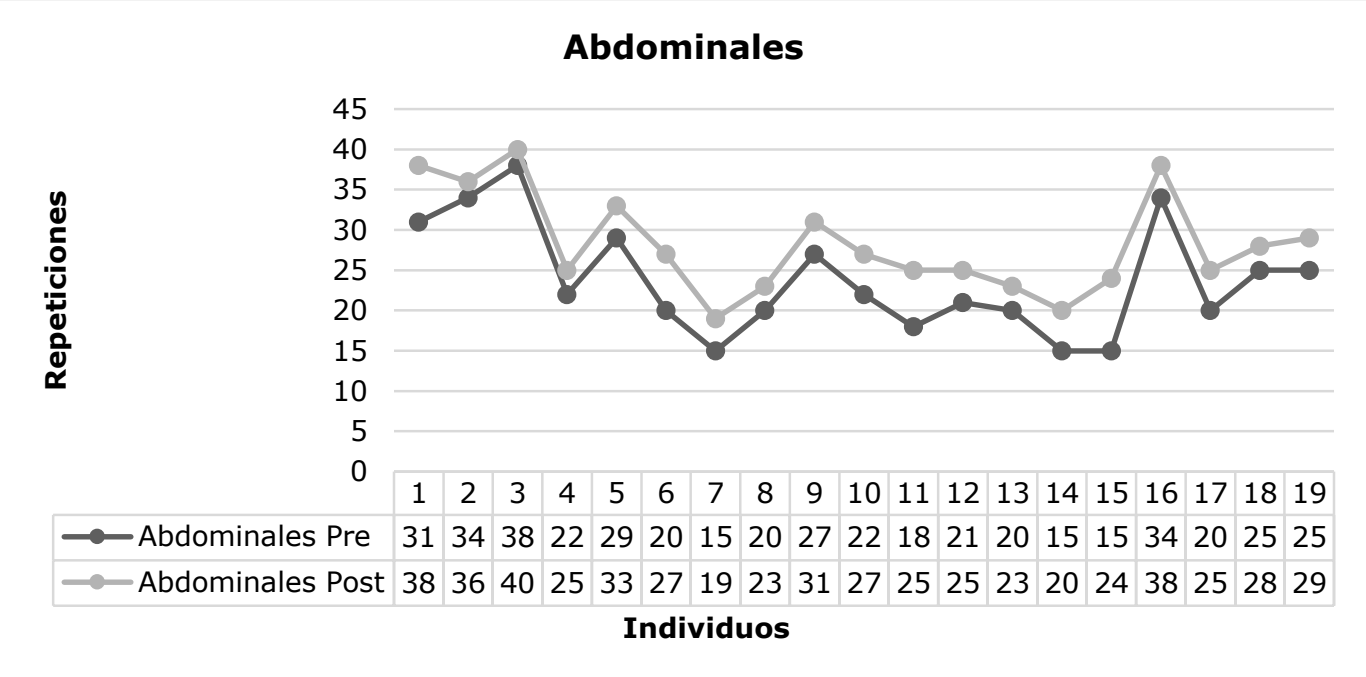

Fuente: elaboración propia.

Teniendo en cuenta los resultados en el ejercicio de abdominales, se puede evidenciar que el individuo con menor nivel de progreso fue el número 3 con un $5 \%$. De igual forma, la persona con mayor nivel de progreso fue el número 15 con $60 \%$.

De acuerdo con la tabla 9, se registra el trabajo realizado por todos los participantes antes y después del programa de entrenamiento de la fuerza. Así, el individuo 11 realizó 18 secuencias de abdominales en el pretest y 25 secuencias de abdominales en la ejecución del postest (tabla 9). 
Tabla 9. Comparación del trabajo al fallo antes y después de la prueba de abdominales

\begin{tabular}{|c|c|c|c|}
\hline & Pretest & \multicolumn{2}{|c|}{ Postest } \\
\hline Individuos & Abdominales & Individuos & Abdominales \\
\hline 19 & 451 & 19 & 536 \\
\hline Promedio & 24 & Promedio & 28 \\
\hline
\end{tabular}

Fuente: elaboración propia.

En la figura 4 se muestra la diferencia entre las repeticiones ejecutadas antes y después de la prueba. Allí se ve claramente que cada individuo mejoró notoriamente gracias a la aplicación del programa de entrenamiento en fuerza. En cuanto al número de secuencias, vale la pena mencionar que, si bien es cierto que algunos participantes aumentaron en forma baja, siempre se evidenció una mejoría (figura 4).

Figura 4. Repeticiones antes y después de la prueba de saltos con sentadillas

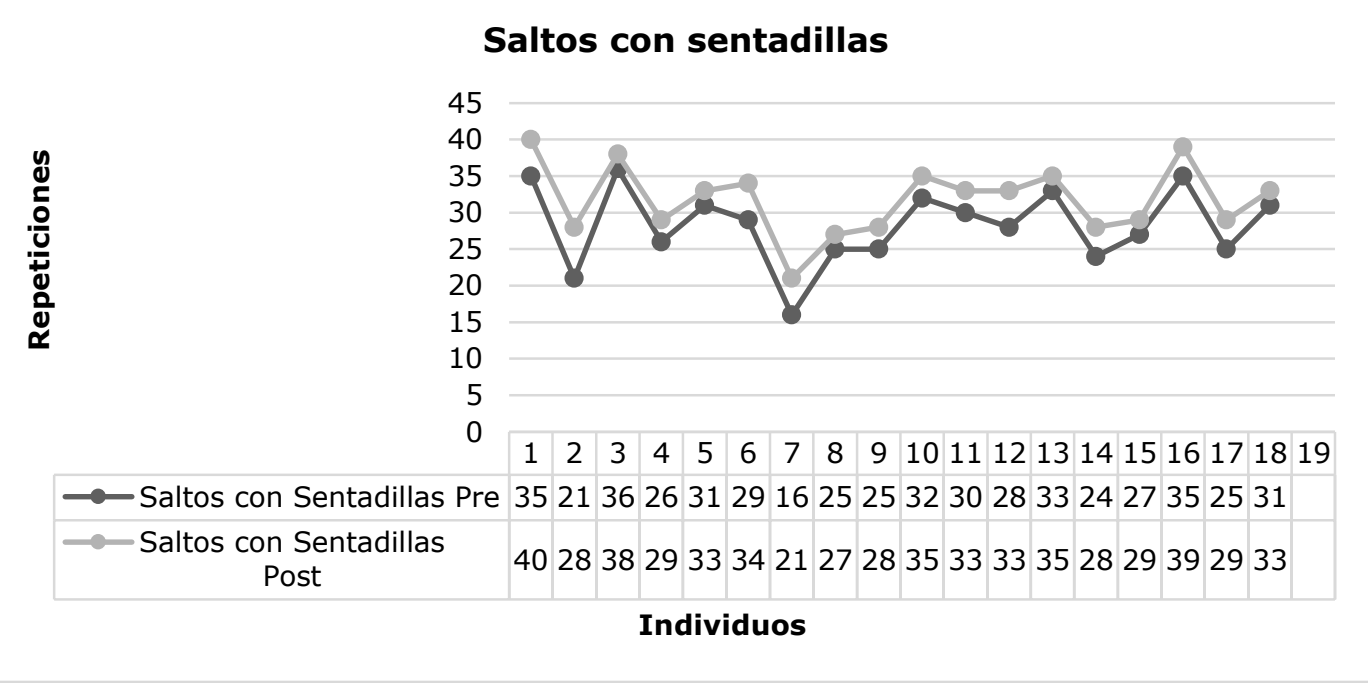

Fuente: elaboración propia.

A lo largo del presente estudio se logró visualizar y comprobar que la fortaleza física en infantes y jóvenes crece en forma ascendente y sostenida, como lo afirma el estudio de Branta et ál. (1984). Allí se 
constata cómo la fortaleza de los músculos se incrementa de forma sostenida durante la infancia en ambos sexos.

Faigenbaum et ál. (2015) sostienen que el entrenamiento de la fuerza ayuda a acondicionar a la juventud practicante del deporte para enfrentar con mejores herramientas las altas demandas musculoesqueléticas del entrenamiento deportivo y la competencia. En el trabajo de campo se evidenció como la mayoría de los niños y adolescentes lograron mantener, sin mayor esfuerzo, su ritmo y su rendimiento; incluso, después de realizar dos entrenamientos de autocarga hasta el fallo y uno anterior a la competencia estabilizador en la que se deseaba conservar las nuevas adaptaciones.

De otro lado, Behringer et ál. (2011) agregan que el mejoramiento de la fuerza muscular y del rendimiento en el desarrollo motriz están respaldadas por la literatura científica, el entrenamiento de fuerza correctamente ordenado e inspeccionado tiene la capacidad de forjar progresos de rendimiento en habilidades motrices, tales como caminar, trotar, correr, saltar, lanzar objetos, entre otros, en la etapa infantil y la adolescencia, lo que puede reflejarse en mejoras de otras capacidades de tipo deportivo.

\section{Conclusiones}

Todos los participantes tenían en promedio 11 años. Todos los promedios de repeticiones, tales como flexiones de rodilla (pretest 21-postest 24); sentadillas (pretest 39-postest 46); abdominales (pretest 23-postest 28) y saltos con sentadilla (pretest 28-postest 32), mejoraron en el postest, algunos de manera muy significativa.

Se observó que todos los jóvenes deportistas que ejecutaron una estrategia para la consolidación de la fuerza y la preparación física mejoraron su 
capacidad de su fuerza. El participante con menor nivel de mejora fue el 18 con $36 \%$. El participante con mayor nivel de mejora fue el número siete con $45 \%$.

Se constata que, con la aplicación de una estrategia de consolidación de la fuerza, todos los participantes mejoraron, bien sea en el número de repeticiones (los casos en donde el postest era mayor al pretest o en el rendimiento de la realización de la actividad.

A lo largo del análisis de la información se descubrió que la estrategia del abordaje de la fuerza fue empleada estrictamente; sin embargo, ocurrió algo inesperado en la realización del postest de las flexiones de rodillas. El individuo 15 realizó las mismas repeticiones en el pretest y en el postest, razón por la cual no hubo progreso y afectó el promedio de secuencias que en el pretest fue de 18 y en el postest 18. Se piensa que, si el participante hubiera realizado el $4 \%$ de sus repeticiones iniciales, lograría 22 secuencias a cambio de las 18 realizadas en el postest, lo que aumentaría el promedio de repeticiones.

Otra conclusión es que un entrenamiento de fuerza en niños y adolescentes mejora el sistema óseo, y a nivel postural hay una mejor adaptación al cuerpo, lo que evita lesiones y brinda fortaleza a los músculos y articulaciones.

La resistencia al fallo y el trabajo de fuerza es útil como orientador para la recolección de datos, cuando no contamos con las herramientas complejas. Asimismo, determinar ejercicios competitivos entre las personas permite un mayor nivel de participación en el deporte.

Finalmente, la investigación señala, con datos estadísticos, que llevar a cabo un programa de fuerza con una carga baja, y en aumento lineal y ascendente, favorece a los prepúberes en su técnica de ejecución, su estado 
físico y por ende en el desempeño del deporte u otra actividad que requiera mayor esfuerzo físico.

\section{Referencias}

Armstrong, N. y Mechelen, W. P. (2013). Paediatric Exercise Science and Medicine. Oxford University Press.

Asmus, A. (1991). Physische und motorische Entwicklung im Kindes und Jugendalter. Bibliothek.

Behringer, M., Vom, A., Matthews, M. y Mester, J. (2011). Effects of Strength Training on Motor Performance Skills in Children and Adolescents: A Meta-Analysis. Pediatric Exercise Science, 23(2), 186-206. https://doi.org/10.1123/pes.23.2.186

Beunen, G. y Malina, R. (2008). Growth and Biologic Maturation: Relevance to Athletic Performance. Semantic Scholar.

Bompa, T. (2003). Periodización. Teoría y metodología del entrenamiento. Hispano Europea.

Branta, C., Haubenstricker, J. y Seefeldt, V. (1984). Age changes in motor skills during childhood and adolescence. Exercice and Sport sciences reviews, 12, 467-520.

Faigembaum, A. (2000). Age and sex related differences and their Implication for resistance exercise. En A. Faigembaum, Essentials of Strength Training and Conditioning (pp. 169-186). Human Kinetics.

Faigembaum, A., Westcott, W., Micheli, L., Outerbridge, A., Long, C. y Zaichkowsky, L. (1996). The effects of strength training and detraining on Children. Strength Cond. Research, 1O(2), 109-114.

Faigenbaum, A., Bush, J., McLoone, R., Kreckel, M., Farrell, A., Ratamess, N. y Kang, J. (2015). Benefits of Strength and Skill-based Training During Primary School Physical Education. Journal of Strength and Conditioning Research, 29(5), 55-62.

Faigenbaum, A., Milliken, L. y Cloutier, G. (2004). Perceived exertion during resistance exercise by children. Perceptual and motor skill, 98, 627-637. https://www.researchgate.net/publication/8564015_Perceived_Exertion_during Resistance Exercise by Children

Revista de Investigación Cuerpo, Cultura y Movimiento ISSN: 2248-4418 | e-ISSN: 2422-474X | DOI: https://doi.org/10.15332/2422474X 
Grosser, M., Brüggemann, P. y Zintl, F. (1989). Alto rendimiento deportivo: planificación y desarrollo. Ediciones Martínez Roca.

Hahn, E. (1988). Entrenamiento con niños. Ediciones Martínez Roca.

Hernández, R., Fernández, C. y Baptista, M. (2014). Metodología de la Investigación. Mc Graw Hill.

Lloyd, R., Faigenbaum, A., Stone, M. y Oliver, J. (2014). Posicionamiento sobre el entrenamiento de fuerza en jóvenes. Consenso Internacional de 2014. Arch Med Deporte, 31(2), 111-124.

https://www.researchgate.net/publication/263444859_Arch_Med_Deporte_2014 312111-124

Malina, R. y Bouchard, C. (2004). Growth, Maturation, and Physical Activity and Edition. Human Kinetics.

Martin, D. (1982). Grundlagen der Trainingslehre Teil 2: Die Planung, Gestaltung und Steuerung des Trainings und das Kinder- und Jugendtrainin (2. ${ }^{\mathrm{a}}$ ed.). Hofmann.

Martin, D., Carl, K. y Lehnertz, K. (1991). Handbuch Trainingslehre. Hofmann.

Organización Mundial de la Salud. (2020, 26 de noviembre). Actividad Física. https://www.who.int/es/news-room/fact-sheets/detail/physical-activity

Watts, K., Jones, T., Davis, E. y Green, G. (2005). Exercise training in obese children and adolescents: current concepts. Sports Medicine, 35(5), 375-392.

Winter, R. (1986). Le fasi sensibili (Parte prima). Rivista di Cultura Sportiva, 6, 8-10. https://scuoladellosport.coni.it/scuoladellosport/biblioteca-e-risorseeditoriali/sds-rivista-di-cultura-sportiva.html

Revista de Investigación Cuerpo, Cultura y Movimiento ISSN: 2248-4418 | e-ISSN: 2422-474X | DOI: https://doi.org/10.15332/2422474X Vol. 11 N. ${ }^{\circ} 2$ | julio-diciembre de 2021 